\title{
AMPLIAÇÃO DA ÁREA DE OCORRÊNCIA DO GATO- -PALHEIRO Leopardus braccatus (Carnivora, Felidae) no estado de Minas Gerais, Brasil
}

\author{
Extension of the area of occurrence of the Pantanal cat Leo- \\ pardus braccatus (Carnivora, Felidae) in the state of Minas \\ Gerais, Brazil
}

Emerson Luiz Campagneri ${ }^{1,3}$ Rua José Mazariolli, 40, 16058-027, Jardim Centenário, Araçatuba, São Paulo, SP E-mail: emersoncampagneri@gmail.com

\author{
ALVES, W.O. ${ }^{2,3}$, BERNARDO,B.G. ${ }^{2,3}$, \\ FERREIRA,A.P.B. ${ }^{2,3,4}$, GONCALVES, M.C.F. ${ }^{2,3}$, ZANZINI, A.C.S. ${ }^{5}$
}

\begin{abstract}
Como citar: CAMPAGNERI, Emerson Luiz et al. Ampliação da área de ocorrência do gato-palheiro Leopardus braccatus (Carnivora, Felidae) no estado de Minas Gerais, Brasil. Revista de Educação Continuada em Medicina Veterinária e Zootecnia do CRMV-SP, São Paulo, v.18, n. 1, 2020. Doi.10.36440/recmvz.v18i1.38028

Cite as: CAMPAGNERI, Emerson Luiz et al. Expansion of the area of occurrence of the pantanal cat Leopardus braccatus (Carnivore, Felidae) in the state of Minas Gerais, Brazil. Journal of Continuing Education in Animal Science of CRMV-SP, São Paulo, v.18, n.1, 2020. Doi.10.36440/recmvz.v18i1.38028
\end{abstract}

\section{Resumo}

O gato-palheiro Leopardus braccatus é um pequeno felídeo não muito maior que um gato doméstico encontrado na Argentina, Brasil, Paraguai e Uruguai. A fragmentação do seu habitat tem sido considerada a maior ameaça para a espécie na maior parte de sua área de distribuição. Nesta nota técnica, apresentamos um novo registro visual da espécie para a porção mais sudoeste do estado de Minas Gerais, Brasil.

Palavras-chave: Leopardus braccatus. Minas Gerais. Registro visual.

\section{Abstract}

The Pantanal cat Leopardus braccatus is a small-sized felid not much larger than a domestic house cat found in Argentina, Brazil, Paraguay and Uruguay. Its habitat fragmentation has been considered the greatest threat to the specie throughout most of its range. In this technical note, it was presented a new visual record of the specie for the more southwestern portion of Minas Gerais state, Brazil.

Keywords: Leopardus braccatus. Minas Gerais. Visual record.

\footnotetext{
1 Engenheiro florestal especialista em Ecologia e Manejo Ambiental

2 Biólogo Analista Ambiental

3 PROJEC Consultoria Ambiental

4 Especialista em Gestão Ambiental

5 Ph.D do Departamento de Ciências Florestais, Universidade Federal de Lavras, Lavras, MG, Brasil
} 


\section{Introdução}

Leopardus braccatus popularmente conhecido no Brasil como gato-palheiro, é um felídeo de pequeno porte (aproximadamente 3,0 kg) (PAGLIA et al., 2012) que ocorre na Argentina, Brasil, Paraguai e Uruguai (BARSTOW; LESLIE JUNIOR, 2012). No Brasil, a espécie é registrada na região centro-oeste e sudeste (Mato Grosso do Sul, sul-sudeste do Mato Grosso, Goiás, Tocantins, sul do Maranhão e Piauí, oeste da Bahia, oeste e noroeste de Minas Gerais e oeste de São Paulo) e na região sul (metade do estado do Rio Grande do Sul) (QUEIROLO et al., 2013).

o Leopardus braccatus é considerada uma espécie distinta de Oncifelis colocolo e do Leopardus colocolo, Wozencraft (2005) reconhece duas subespécies de L. braccatus. Leopardus braccatus braccatus e Leopardus braccatus munoai. A primeira habita a região central da América do SUI (BARSTOW; LESLIE JUNIOR, 2012) e no Brasil a espécie é registrada nos estados do Maranhão, Piauí, Tocantins, Goiás, Mato Grosso, Mato Grosso do Sul, Bahia, Minas Gerais e São Paulo (QUEIROLO et al., 2013). A segunda ocorre unicamente no Uruguai e no estado do Rio Grande do Sul, Brasil (BARSTOW; LESLIE JUNIOR, 2012).

A fragmentação e destruição de habitats nas regiões em que Leopardus braccatus braccatus ocorre, são as principais ameaças e causas do declínio populacional (CHEIDA et al., 2006; OLIVEIRA; CASSARO, 2005), outras potenciais ameaças são o atropelamento em rodovias e zoonoses transmitidas por animais domésticos. No estado de Minas Gerais a espécie é considerada Em Perigo (COPAM, 2010). No Brasil é considerada Vulnerável (BRASIL, 2014) e internacionalmente é considerada como Quase Ameaçada (LUCHERINI et al., 2016).

Nesta nota técnica são apresentados um registro visual inédito de Leopardus braccatus braccatus para o município de Itapagipe, extremo sudoeste do estado de Minas Gerais e um mapa atualizado com base na literatura (BAGNO et al., 2004; COURTENAY, 2002; MACHADO; DRUMMOND; PAGLIA, 2008; NASCIMENTO; POMPEU; PASSAMANI, 2016; QUEIROLO et al., 2013; SILVEIRA; PAULA; RODRIGUES, 2008) sobre a atual distribuição da espécie no Estado.

0 registro visual da espécie ocorreu em 9 de maio de 2016 por volta das 17:30 horas, durante um trabalho de monitoramento de fauna na zona rural do município de Itapagipe, Minas Gerais. 0 registro foi obtido nas coordenadas geográficas definidas por $19^{\circ} 53^{\prime} 24^{\prime \prime}$ de latitude Sul e $49^{\circ} 42^{\prime}$ 08" de longitude Oeste, a uma altitude média de 420 metros. A região onde ocorreu o registro está inserida no Bioma Cerrado, especificamente na Bacia Hidrográfica do Rio Grande, extremo sudoeste do estado de Minas Gerais, divisa com o estado de São Paulo.

A equipe circulava com veículo motorizado em uma estrada não pavimentada margeada por cultura de cana-de-açúcar e fragmento de Floresta Estacional Semidecídua quando se deparou com uma fêmea com filhote que se deslocava entre o fragmento florestal e a área de cultivo de canade-açúcar. Durante o contato visual, a fêmea e o filhote pararam por alguns segundos em frente ao veículo, tempo suficiente para o registro fotográfico dos espécimes (Figura 1). 
Figura 1. Registros fotográficos de Leopardus braccatus braccatus
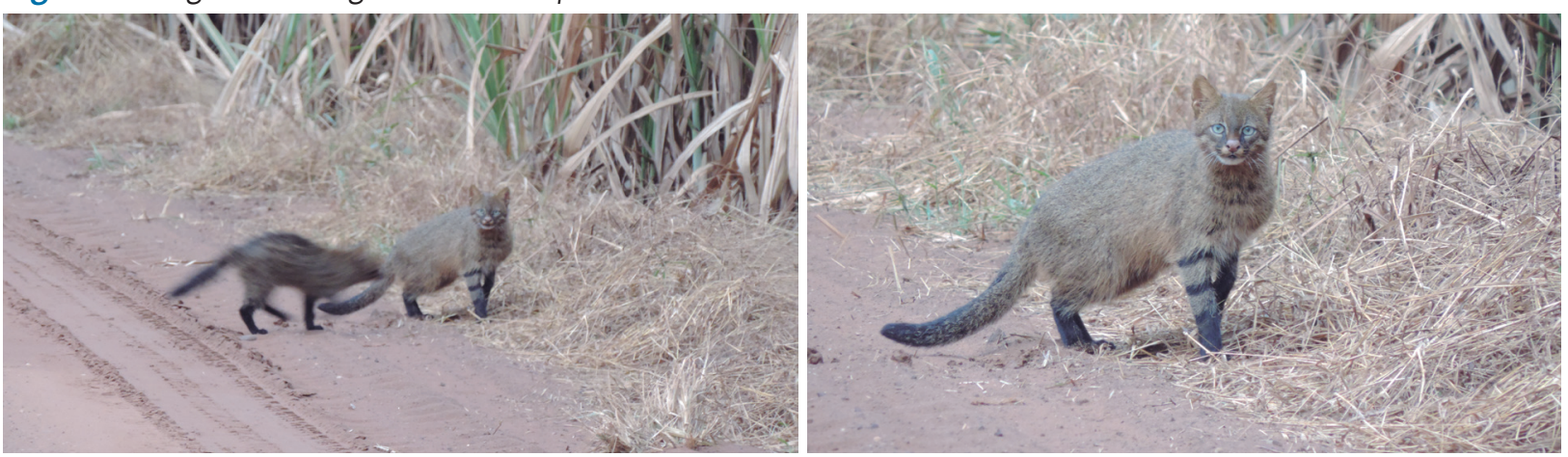

T Fonte: Autores (2016).

Legenda 1. Registros fotográficos de Leopardus braccatus braccatus em estrada não pavimentada margeada por Floresta Estacional Semidecídua e cultura de cana-de-açúcar na zona rural do município de Itapagipe, extremo sudoeste do estado de Minas Gerais, Brasil, nas coordenadas geográficas definidas por $19^{\circ} 53^{\prime} 24^{\prime \prime}$ de latitude Sul e $49^{\circ} 42^{\prime} 08^{\prime \prime}$ de longitude Oeste

Esta nova localidade de registro de L. b. braccatus representa uma ampliação da área de distribuição da subespécie em aproximadamente $500 \mathrm{~km}$ do Parque Nacional da Serra da Canastra, representando o limite mais ocidental de ocorrência de L. b. braccatus no território do estado de Minas Gerais, Brasil (Figura 2).

Figura 2. Distribuição geográfica de Leopardus braccatus braccatus no estado de Minas Gerais, Brasil

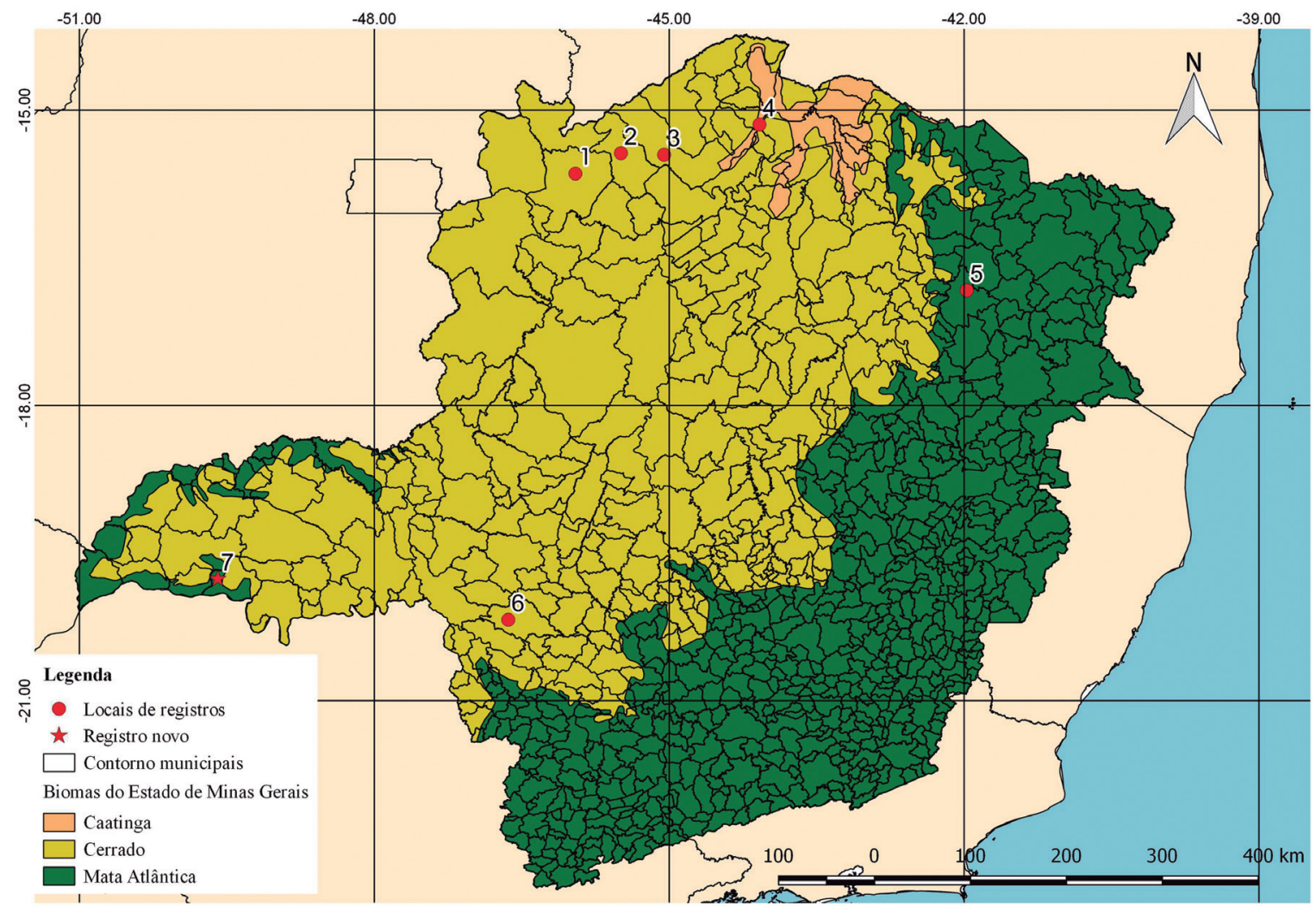

T Fonte: Adaptado do IBGE (2010); BRASIL (2006).

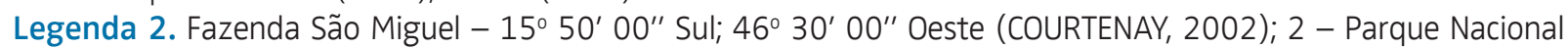
Grande Sertão Veredas - 15 $18^{\prime} 21^{\prime \prime}$ Sul; $45^{\circ} 37^{\prime} 04^{\prime \prime}$ Oeste (BAGNO et al., 2004); 3 - RPPN Porto Cajueiro - $15^{\circ} 29^{\prime}$

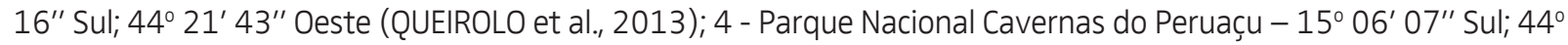

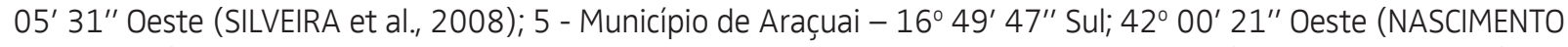

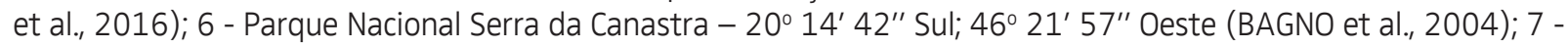
Município de Itapagipe - 19053'24" Sul; 49042'08" Oeste

Nota: Os círculos em vermelhos indicam a ocorrência da espécie em localidades pesquisadas na literatura. A estrela vermelha (referência 7) refere-se ao novo registro obtido no município de Itapagipe, extremo sudoeste de Minas Gerais, Brasil, nas

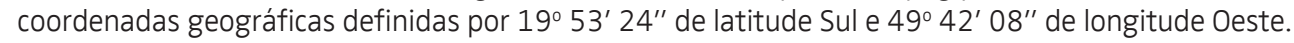


A presente nota técnica constitui uma importante referência para a ampliação da área de ocorrência de Leopardus braccatus braccatus no território nacional e, especificamente, no território do estado de Minas Gerais, Brasil. Em toda sua área de ocorrência, essa espécie não costuma ser alvo direto de esforços de pesquisa e os registros de sua ocorrência são obtidos em projetos que visam levantamentos da comunidade de mamíferos de médio e grande porte como um todo (BAGNO et al., 2004; SILVEIRA; PAULO; RODRIGUES, 2008). Também não existem levantamentos abrangentes avaliando o número de L. b. braccatus existentes na natureza (BARSTOW; LESLIE JUNIOR, 2012), no entanto os poucos estudos já realizados sugerem que a espécie é mais rara que os demais felídeos de porte semelhante (INDRUSIAK; EIZIRIK, 2003), ocorrendo em densidades tipicamente baixas de 0,01 a 0,05 indivíduos / $\mathrm{km}^{2}$, chegando a densidades um pouco maiores de 0,1 a 0,2 indivíduos/ $\mathrm{km}^{2}$ em locais onde sua ocorrência é relativamente comum (OLIVEIRA et al., 2010; OLIVEIRA, 2011).

As principais ameaças para a espécie são a fragmentação da paisagem (CHEIDA et al., 2006; OLIVEIRA; CASSARO, 2005), o atropelamento em estradas (BAGNO et al., 2004) e as zoonoses transmitidas por animais domésticos (QUEIROLO et al., 2013).

As ações a serem desencadeadas para resguardar a espécie incluem a manutenção dos fragmentos florestais na paisagem e a implantação de medidas preventivas contra os eventos de atropelamentos e a caça retaliatória.

\section{Referências}

BAGNO, M. A. et al. Notes on the natural history and conservation status of pampas cat, Oncifelis colocolo, in the Brazilian Cerrado. Mammalian, New York, v. 68, n. 1, p. 75-79, Jul. 2004.

BARSTOW, A. L.; LESLIE JUNIOR, D. M. Leopardus braccatus (Carnivora: Felidae). Mammalian Species, New York, v. 44, n. 891, p. 16-25, Apr. 2012.

BRASIL. Portaria no 444, de 17 de dezembro de 2014. Diário Oficial da União: seção 1, Brasília, DF, p. 121, 18 dez. 2014.

BRASIL. Ministério do Meio Ambiente. Secretaria de Biodiversidade e Florestas. Mapa de Cobertura vegetal do Bioma Cerrado. Planaltina: Embrapa Cerrados; Uberlândia: Universidade Federal de Uberlândia; Goiânia: Universidade Federal de Goiás, 2006. Disponível em: http://mapas.mma.gov.br/ geodados/brasil/vegetacao/vegetaca02002/cerrado/mapas_pdf/vegetacao/mosaico/mosaico_ cobvegA0.pdf. Acesso em: 3 jan. 2020.

CHEIDA, C. C. et al. Ordem carnívora. In: REIS, N. R. et al. (Ed.). Mamíferos do Brasil. Londrina: Ed. UEL, 2006. p. 231-275.

COPAM - CONSELHO DE POLÍTICA AMBIENTAL. Deliberação Normativa COPAM no 147, de 30 de abril de 2010. Aprova a Lista de Espécies Ameaçadas de Extinção da Fauna do Estado de Minas Gerais. Diário do Executivo do Estado de Minas Gerais, Belo Horizonte, 4 maio 2010. Disponível em: http://www.ief.mg.gov.br/. Acesso em: 3 jan. 2020.

COURTENAY, O. A new record of pampas cat, Lynchailurus braccatus, in Brazil. Mammalia, Paris, v. 66, n. 2, p. 295-297, Jan. 2002.

IBGE - INSTITUTO BRASILEIRO DE GEOGRAFIA E ESTATÍSTICA. Atlas nacional do Brasil: vegetação: biomas. Rio de Janeiro: IBGE. IBGE, 2010. Disponível em: https://geoftp.ibge.gov.br/atlas/nacional/ atlas nacional do brasil 2010/2 territorio e meio ambiente/atlas nacional do brasil 2010 pagina 89 biomas.pdf. Acesso em: 14 jan. 2020. 
INDRUSIAK, C.; EIZIRIK, E. Carvívoros. In: FONTANA, C. S.; BENCKE, G. A.; REIS, R. E. (Ed.). Livro vermelho da fauna ameaçada de extinção no Rio Grande do Sul. Porto Alegre: EDIPUCRS, 2003. p. 507-533.

LUCHERINI, M. et al. Pampas cat: Leopardus colocolo. The IUCN Red List of Threatened Species, e.T15309A97204446, 2016. Disponível em: http://dx.doi.org/10.2305/IUCN.UK.2016-1.RLTS. T15309A97204446.en. Acesso em: 23 nov. 2019.

MACHADO, A. B. M.; DRUMMOND, G. M.; PAGLIA, A. P. (Ed.). Livro vermelho da fauna brasileira ameaçada de extinção. Brasília, DF: MMA, 2008. 2v. 1420p.

NASCIMENTO, F. O.; POMPEU, P. S.; PASSAMANI, M. Range extension of the pantanal cat Leopardus braccatus (Carnivora, Felidae) in a cerrado-caatinga-atlantic forest ecotone, Brazil. Mastozoologia Neotropical. Mendoza, v. 23, n. 1, p. 171-177, July 2016.

OLIVEIRA, T. G. de et al. Ocelot ecology and its effect on the small-felid guild in the lowland neotropics. In: MACDONALD, D. W.; LOVERIDGE, A. J. (Ed.). Biology and conservation of the wild felids. Oxford: Oxford University Press, 2010. p. 559-580.

OLIVEIRA, T. G. de. Ecologia e conservação de pequenos felinos no Brasil e suas implicações para o manejo. Tese (Doutorado em Ecologia, Conservação e Manejo da Vida Silvestre) - Universidade Federal de Minas Gerais, Belo Horizonte, 2011.

OLIVEIRA, T. G.; CASSARO, K. Guia de felinos do Brasil. São Paulo: Instituto Pró-Carnívoros, 2005. 80 p.

PAGLIA, A. P. et al. Lista anotada dos mamíferos do Brasil. 2. ed. Arlington: Conservation International, 2012. 76 p.

QUEIROLO, D. et al. Avaliação do risco de extinção do Gato-palheiro Leopardus colocolo (Molina, 1782) no Brasil. Biodiversidade Brasileira, Brasília, v. 3, n. 1, p. 91-98, 2013.

SILVEIRA, L.; PAULA, R. C.; RODRIGUES, F. H. G. Oncifelis colocolo Molina, 1782. In: MACHADO, A. B. M.; DRUMMOND, G. M.; PAGLIA, A. P. (Ed.). Livro vermelho da fauna brasileira ameaçada de extinção. Brasília, MMA, Fundação Biodiversitas, 2008. v.2, p.791-792.

WOZENCRAFT, W. C. Order Carnivora. In: WILSON, D. E.; REEDER, D. M. (Ed.). Mammal species of the world: a taxonomic and geographic reference. 3. ed. Baltimore: The Johns Hopkins University Press, 2005. p. 532-628. 


\section{Referências}

ACHAVAL, F.; OLMOS, M. C. Mamíferos de la República Oriental del Uruguay: guia fotográfica. 2. ed. Montevideo: Zonolibro Indústria Gráfica, 2007. 216 p.

BRESSAN, P. W.; KIERULFF, M. C. M.; SUGIEDA, A. M. Fauna ameaçada de extinção no Estado de São Paulo: vertebrados. São Paulo: Fundação Parque Zoológico de São Paulo: Secretaria do Meio Ambiente, 2009. 648 p.

ESPINOSA, C. C. et al. Medium-and large-sized mammals in a steppic savanna area of the Brazilian Pampa: survey and conservation issues of a poorly known fauna. Brazilian Journal of Biology, São Carlos, v. 76, n. 1, p. 73-79, fev. 2016.

FONTANA, C. S. et al. (Org.). Livro Vermelho da Fauna ameaçada de extinção no Rio Grande do Sul. Porto Alegre: EDIPUCRS, 2003. 632 p.

INSTITUTO ESTADUAL DE FLORESTAS. Portal meioambiente.mg. Belo Horizonte, 2016. Disponível em: http://www.ief.mg.gov.br/. Acesso em: 22 dez. 2016.

LUCHERINI, M.; VIDAL, E. L.; MERINO, M. J. How rare is the rare Andean cat? Mammalia, Paris, v. 72, n. 1, p. $95-101,2008$.

MAMEDE, S. B.; ALHO, C. J. R. Impressões do Cerrado \& Pantanal: subsídios para a observação de mamíferos silvestres não voadores. 2. ed. Campo Grande: Ed. UFMS, 2008. 208 p.

MARQUES, A. A. B. et al. (Org.). Lista de Referência da Fauna ameaçada de extinção do Rio Grande do Sul: Decreto no 41.672 de 11 de junho de 2002. Porto Alegre: FZB/MCT-PUCRS, 2002. 52 p.

OLIVEIRA, T. F. Neotropical cats: ecology and conservation. São Luiz: EDUFMA, 1994. 220 p.

PEREIRA, J. et al. Leopardus colocolo. IUCN Red List of Threatened Species, version 2010.4, 2008.

PEREIRA, J.; VARELA, D.; FRACASSI, N. Pampas cat in Argentina: is it absent from the Pampas? Cat News, V. 36, p. 20-22, jan. 2002.

SILVA, F. Mamíferos silvestres do Rio Grande do Sul. 2. ed. Porto Alegre: Fundação Zoobotânica do Rio Grande do Sul, 1994. 255 p.

SUNQUIST, M.; SUNQUIST, F. Wild cats of the world. Chicago: University of Chicago Press, 2002. 416 p.

WALKER, S. R. et al. Diets of three species os Andean carnivores in high-altitude deserts of Argentina. Journal os Mammalogy, Lawrence, v. 88, n. 2, p. 519-525, Apr. 2007. 